\title{
Abundance analysis of barium stars
}

\section{Guochao Yang ${ }^{1,2}$, Yanchun Liang ${ }^{1}$, Wenyuan Cui ${ }^{2}$ and Bo Zhang ${ }^{2}$}

${ }^{1}$ Key Laboratory of Optical Astronomy, National Astronomical Observatories, Chinese Academy of Sciences, Beijing 100012, China, email: gcyang@bao.ac.cn,ycliang@bao.ac.cn

${ }^{2}$ Department of Physics, Hebei Normal University, Shijiazhuang 050024, China

\begin{abstract}
We obtained the chemical abundances of more than 20 Barium (Ba) stars by analysing high resolution echelle spectra. They show obvious overabundances of neutron capture process elements, such as Y, Zr, Ba, La and Eu. Their abundance pattern can be explained by binary accretion through stellar wind, where the Ba stars have accreted the ejecta from the companion stars which were in AGB stages at that time and now evolve as white dwarfs.
\end{abstract}

Keywords. Stars: abundances, Stars: atmospheres, Stars: chemically peculiar, Stars: late-type

Barium ( $\mathrm{Ba}$ ) stars are $\mathrm{G}$ to $\mathrm{K}$ giants with abnormally strong lines of $s$-process elements, as well as enhanced $\mathrm{CH}, \mathrm{CN}$, and $\mathrm{C}_{2}$ molecular bands (Bidelman \& Keenan (1951). The high resolution and high $S / N$ ratios Echelle spectra of more than $20 \mathrm{Ba}$ stars were obtained by using the $2.16 \mathrm{~m}$ telescope of $N A O C$ in Xinglong station. Their abundances of $\mathrm{O}, \mathrm{Na}, \mathrm{Mg}, \mathrm{Al}, \mathrm{Si}, \mathrm{Ca}, \mathrm{Sc}, \mathrm{Ti}, \mathrm{V}, \mathrm{Cr}, \mathrm{Mn}, \mathrm{Ni}$ are similar to the solar abundances. The neutron capture ( $n$-capture) process elements Y, Zr, Ba, La, Eu show obvious overabundance relative to the Sun generally (Fig. 1 for an example). Their overabundant heavy-element patterns can be explained from a theoretical model of wind accretion in binary system (Liang et al. (2000), Liu et al. (2000)), where they accreted the ejecta from the companions at AGB stages (now evolve as white dwarfs) through stellar winds. This work has been supported by the NSFC under Grant No. 11273026, 10933001, 10973006, 10973015, 11073026, 11273011, U1231119 and 11003002; the Science Foundation of Hebei Normal University under Grant No. L2009Z04; the Natural Science Foundation of Hebei Province under Grant No. A2011205102, and the Program for Excellent innovative Talents in University of Hebei Province under Grant No. CPRC034.
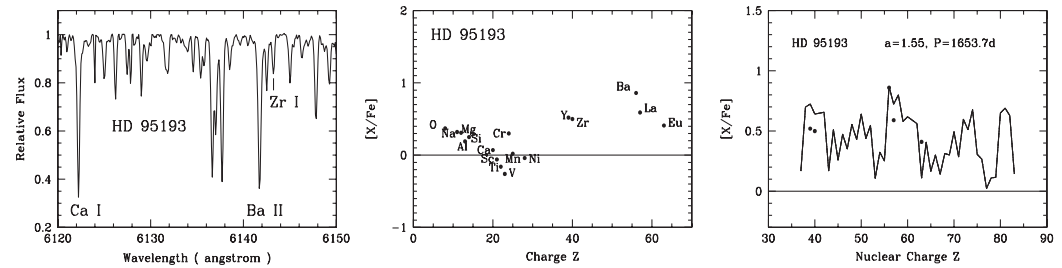

Figure 1. An example: A part of spectrum of HD 95193 (left), its observed abundance pattern (middle), the predicted heavy-element abundances from the wind accretion model (right, "a" indicates the level of overabundance of AGB star, "P" refers to the orbital period of binary).

\section{References}

Bidelman, W. K., \& Keenan, P. C. 1951, APJ, 114, 473

Liang, Y. C., Zhao, G., \& Zhang, B. 2000, A\&A, 363, 555

Liu, J. H., Zhang, B., Liang, Y. C., \& Peng, Q. H. 2000, A\&A, 363, 660 